\title{
Tilted X-Ray Holography of Magnetic Bubbles in MnNiGa Lamellae
}

\author{
Luke A. Turnbull,* Max T. Birch, Angus Laurenson, Nick Bukin, Erick O. Burgos-Parra, Horia Popescu,
} Murray N. Wilson, Aleš Stefančič, Geetha Balakrishnan, Feodor Y. Ogrin, and Peter D. Hatton

Cite This: ACS Nano 2021, 15, 387-395

ABSTRACT: Nanoscopic lamellae of centrosymmetric ferromagnetic alloys have recently been reported to host the biskyrmion spin texture; however, this has been disputed as the misidentication of topologically trivial type-II magnetic bubbles. Here we demonstrate resonant soft X-ray holographic imaging of topological magnetic states in lamellae of the centrosymmetric alloy $\left(\mathrm{Mn}_{1-x} \mathrm{Ni}_{x}\right)_{0.65} \mathrm{Ga}_{0.35}(x=0.5)$, showing the presence of magnetic stripes evolving into single core magnetic bubbles. We observe rotation of the stripe phase via the nucleation and destruction of disclination defects. This

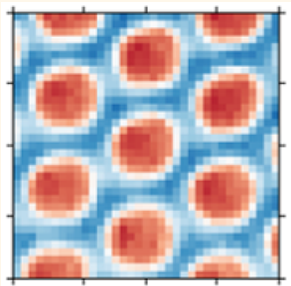

Normal Incidence

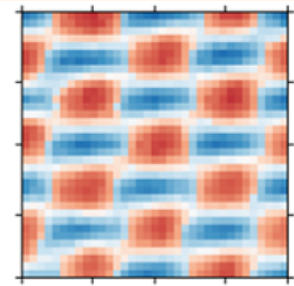

Tilted Image

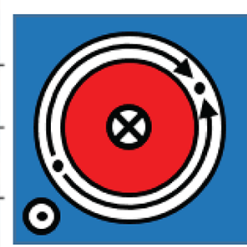

Non-topological Bubble indicates the system behaves as a conventional uniaxial ferromagnet. By utilizing the holography with extended reference by autocorrelation linear differential operator (HERALDO) method, we show tilted holographic images at $30^{\circ}$ incidence confirming the presence of type-II magnetic bubbles in this system. This study demonstrates the utility of $\mathrm{X}$-ray imaging techniques in identifying the topology of localized structures in nanoscale magnetism.

KEYWORDS: $\mathrm{MnNiGa}$, X-ray holography, biskyrmion, topological magnetism, type-II bubble

$\mathrm{T}$ he study of nanoscopic topological magnetization textures such as skyrmions, merons, and domain walls has led to the discovery of emergent physical phenomena, which indicates their potential applications in advanced spintronic devices. ${ }^{1-4}$ Chiral magnets, such as the B20 helimagnets, commonly host skyrmions due to competition between the Dzyaloshinskii-Moriya interaction (DMI) and ferromagnetic exchange interaction. ${ }^{5}$ However, topologically nontrivial spin textures have also been reported in centrosymmetric magnets, which lack DMI, arising due to long-range dipolar forces and uniaxial magnetocrystalline anisotropy. ${ }^{6}$

The formation of magnetic bubble domains in such uniaxial magnets is well documented. Thiele showed in 1969 that magnetic stripe domains will spontaneously form in lamellae of arbitrary thicknesses, if the uniaxial anisotropy coefficient, $K_{w}$ exceeds the stray field energy coefficient, $K_{d}{ }^{7}$ This can be represented by the inequality $\frac{K_{u}}{K_{d}}>1 .{ }^{8}$ Under an increasing out-of-plane magnetic field, these stripes contract and rupture, ultimately forming cylindrical bubble domains. If the domain wall of a bubble has a singular continuous winding, then it is topologically identical to a skyrmion and known as a type-I bubble (Figure 1a). ${ }^{9}$ However, the lack of a symmetry breaking interaction, such as DMI, in centrosymmetric magnets means that there is no global chirality imposed for spin textures and allows structures such as Bloch lines to stabilize within domain walls, forming other topological configurations. ${ }^{10} \mathrm{~A}$ bubble formed of two half circular domain walls with opposite chirality, connected at Bloch lines, has no global winding and is known as a type-II bubble (Figure 1c). ${ }^{11}$ These differences can be classified by the topological winding number, $N=(1 /$ $4 \pi) \int \mathbf{m} \cdot\left(\partial_{x} \mathbf{m} \times \partial_{y} \mathbf{m}\right) \mathrm{d} x \mathrm{~d} y$, which counts the number of times the mapping of a spin configuration, $\mathbf{m}$, wraps the unit sphere. Type-I bubbles are defined by $N=1$, whereas topologically trivial states such as type-II bubbles and stripes exhibit $N=0$. Such differences carry profound consequences for the energetics and transport dynamics of the respective topological classes. ${ }^{12}$ The intense research efforts surrounding topological magnetism has also led to reports of more exotic objects in centrosymmetric systems, such as the biskyrmion, a bound pair of skyrmions with opposing chirality, characterized by $N=2$ (Figure 1e). ${ }^{13}$

Received: September 2, 2020

Accepted: October 23, 2020

Published: October 29, 2020 

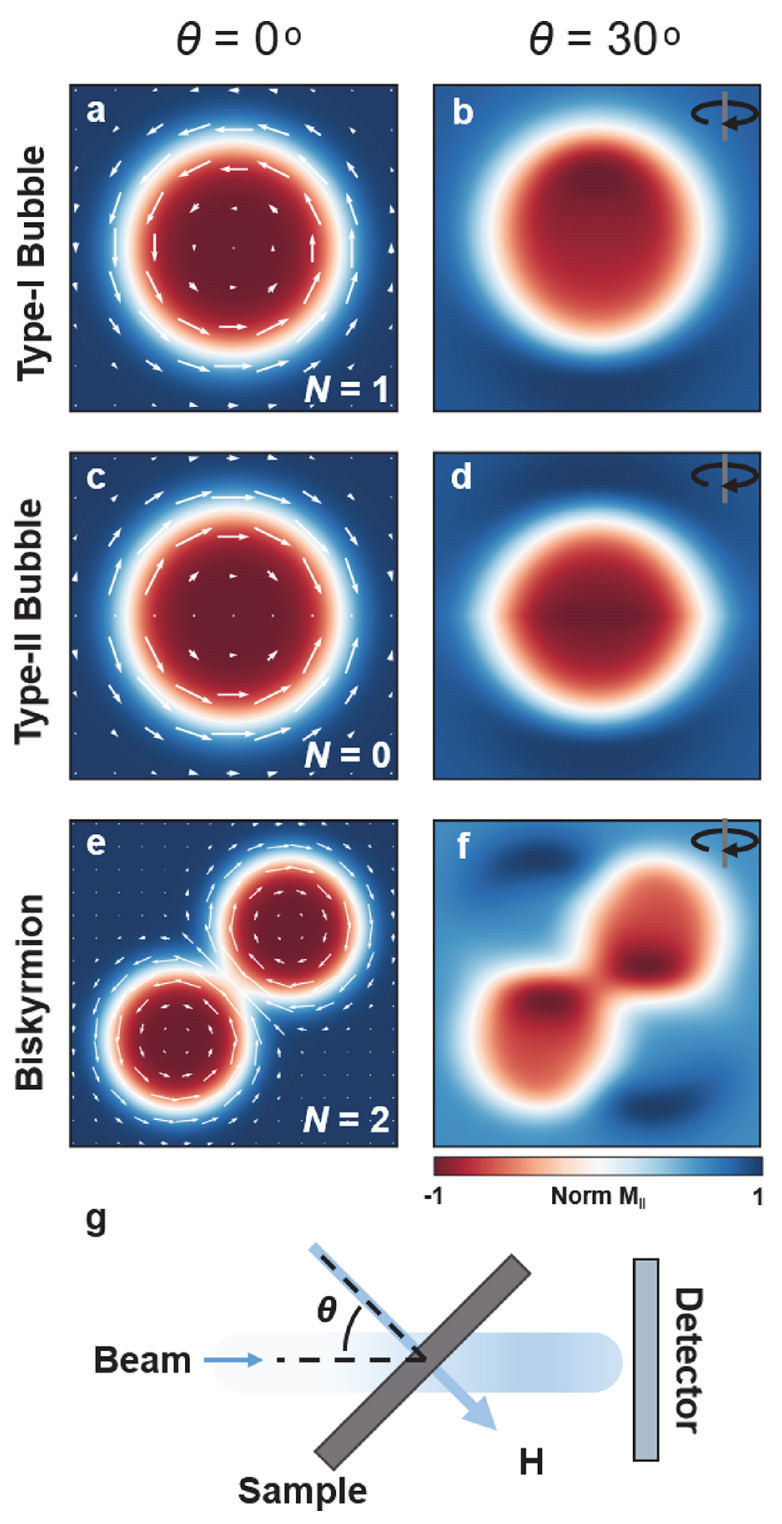

Figure 1. X-ray imaging projections of magnetic bubbles. (a, b) Xray imaging projection of a type-I magnetic bubble at normal incidence and tilted $30^{\circ}$ about the vertical axis, respectively. Arrows indicate the in-plane magnetization components at normal incidence. (c, d) Equivalent projections of a type-II bubble. (e, f) Equivalent projections of a biskyrmion. (g) Schematic of the offnormal transmission scattering geometry.

The properties of nanoscopic magnetization textures are dominated by local features, such as dislocations and defects in the magnetic state, which cannot be examined via reciprocal space measurements, compelling the use of real-space imaging techniques to fully characterize their behavior. ${ }^{14,15}$ Electronbased transmission imaging techniques, including Lorentz transmission electron microscopy (LTEM) ${ }^{16,17}$ and electron holography, ${ }^{18,19}$ are commonly used to map topological spin textures in thin film samples, primarily due to their high spatial resolution of $2-5 \mathrm{~nm} .{ }^{20}$ Electrons are sensitive to components of the magnetic flux density in the plane normal to their incident direction, creating a depth-averaged projection of the in-plane field following the entire path of the electrons, including stray fields above and below the sample. This approach enabled real-space imaging of magnetic skyrmions. ${ }^{21}$ In 2014, the observation of biskyrmions in nanoscopic lamellae of the centrosymmetric systems $\mathrm{La}_{2-2 x} \mathrm{Sr}_{1+2 x} \mathrm{Mn}_{2} \mathrm{O}_{7} \quad(x=$ $0.315)(\mathrm{LSMO})^{22}$ and $\left(\mathrm{Mn}_{1-x} \mathrm{Ni}_{x}\right)_{0.65} \mathrm{Ga}_{0.35} \quad(x=0.5)$ $(\mathrm{MnNiGa})^{23,24}$ was reported using LTEM. However, it was subsequently proposed that reports of biskyrmions had misidentified topologically trivial type-II magnetic bubbles, due to the similarity of their respective LTEM projections. ${ }^{25,26}$

Resonant X-ray holography is a complementary transmission imaging technique, which encodes a sample's magnetization components parallel with the incident X-ray beam, by utilizing the element-specific X-ray magnetic circular dichroism effect. $^{27,28}$ As this method is directly sensitive to the magnetization components of a texture, the twin core structure of a biskyrmion would be easily distinguished from the single core type I or type II bubbles. Holography techniques capture the interference of a reference wave with a coherent beam of circularly polarized light, scattered through the sample, ${ }^{29}$ which is then Fourier transformed to reconstruct a real space projection of the out-of-plane magnetization. The holography with extended reference by autocorrelation linear differential operator (HERALDO) implementation uses the sharp edges of an extended slit to form the reference wave, ${ }^{30-33}$ which produces images with a resolution set by the sharpness of the slit edge and makes it possible to tilt a sample relative to the incoming beam, while maintaining the reference wave intensity (Figure 2). ${ }^{34}$ Imaging at off-normal incidence allows in-plane components of the magnetization to be examined, from which the chiralities of topological magnetic textures can be inferred (Figure 1g). Figure 1 shows the expected X-ray imaging projection of type-I and -II bubbles and biskyrmions at normal incidence and $30^{\circ}$ off-normal incidence. While the projections at normal incidence for type-I and type-II bubbles are identical, they can be distinguished by imaging at off-normal angles. The singular winding of the type-I bubble creates an anisotropic response due to domain wall components pointing in opposite directions about the core. The type-II bubble is symmetric about the horizontal axis and therefore has an isotropic response when tilted about the vertical axis.

In this study we present resonant X-ray holographic images of $\mathrm{MnNiGa}$ lamellae, demonstrating that the system behaves as a conventional uniaxial ferromagnet, hosting magnetic stripes, which rupture into single core bubbles. We observe field induced rotation of the stripe phase via the nucleation and destruction of disclination defects. The HERALDO method is used to demonstrate that the magnetic bubbles have a type-II structure, by performing off-normal imaging, providing further evidence to support claims that earlier reports of biskyrmions had misidentified these topologically trivial bubbles.

\section{RESULTS/DISCUSSION}

Holographic images of a disordered bubble state produced at the $\mathrm{Mn}-\mathrm{L}_{3}(637 \mathrm{eV})$ and $\mathrm{Ni}-\mathrm{L}_{3}(852 \mathrm{eV})$ absorption edges are shown in Figure $3 a, b$. Domains of blue and red represent outof-plane magnetization toward and away from the reader, while white regions indicate net magnetization entirely in the plane of the sample. The energies of both absorption edges were confirmed by performing small-angle X-ray scattering energy scans. Figure $3 \mathrm{c}, \mathrm{d}$ shows the corresponding X-ray absorption spectra and equivalently normalized magnetic scattering intensity as a function of the energy, for a remanent stripe state at $300 \mathrm{~K}$. Resonant X-ray techniques allow element specific magnetic imaging, and the image similarity exhibited in Figure $3 \mathrm{a}, \mathrm{b}$ indicates the same magnetic structure, within resolution limits. This demonstrates equivalent magnetic 


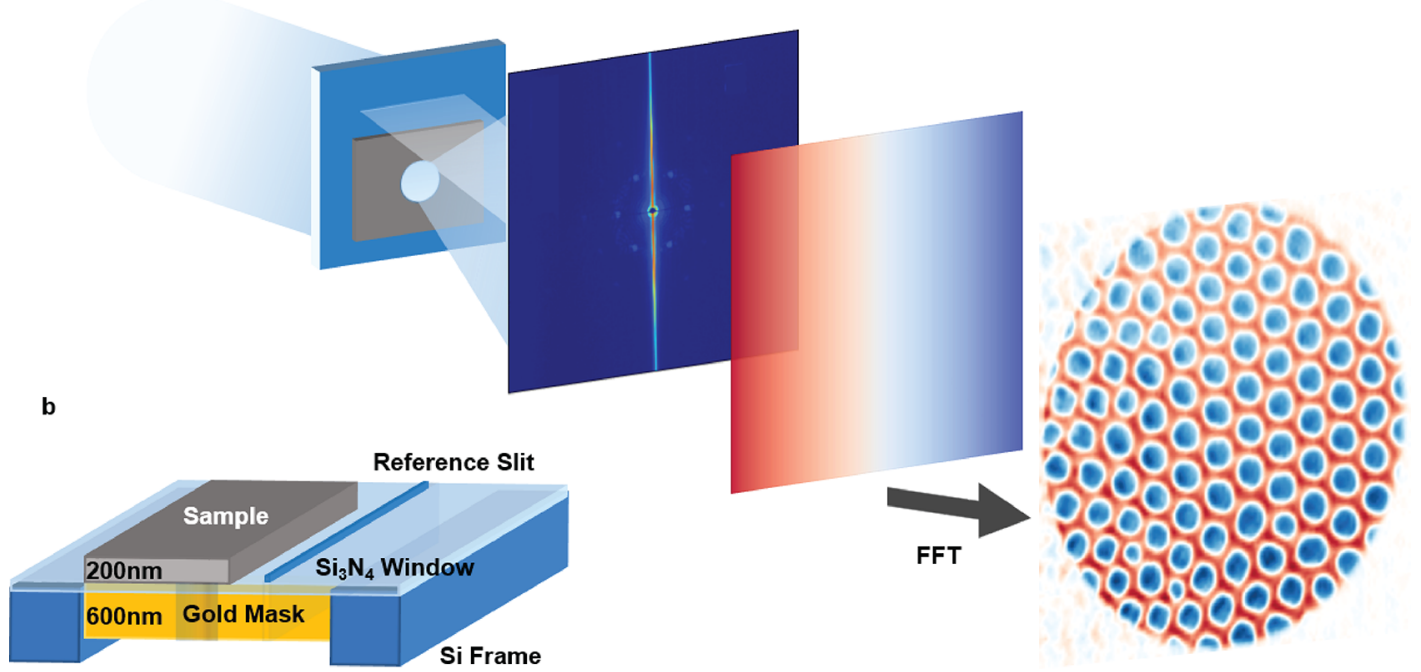

Figure 2. Schematic of the HERALDO imaging process and sample configuration. (a) HERALDO reconstruction process, showing coherent $\mathrm{X}$-rays incident on the sample and reference slit, which diffract and interfere to form a hologram on the CCD. A differential filter in the direction of the slit is then applied, followed by a Fourier transform to reconstruct the real space image, an example of which is shown on the right. (b) HERALDO sample configuration, showing a lamella mounted onto a $\mathrm{Si}_{3} \mathrm{~N}_{4}$ window. A gold mask is used to form the sample aperture and reference slit.
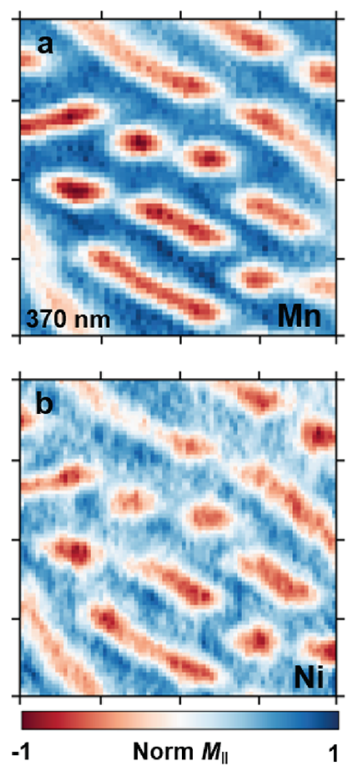
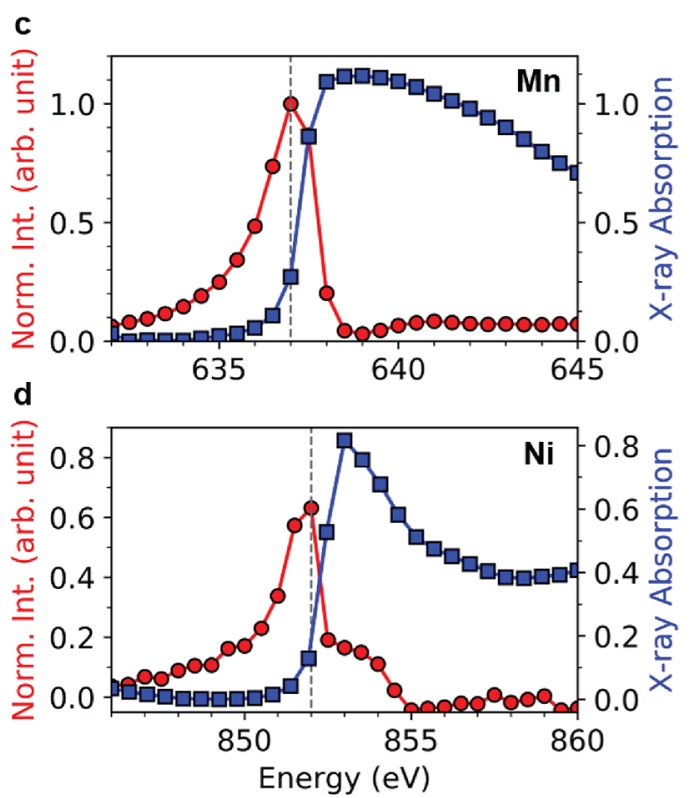

Figure 3. Element specific imaging and energy spectra. (a, b) X-ray holographic images of the same disordered bubble state at $300 \mathrm{~K}$ and 100 $\mathrm{mT}$, produced at the $\mathrm{Mn}-\mathrm{L}_{3}$ and $\mathrm{Ni}-\mathrm{L}_{3}$ edges, respectively. Scale bar ticks represent $370 \mathrm{~nm}$. (c) X-ray absorption spectra $\left(-\ln \left(I / I_{0}\right)\right.$, where $I$ is the transmitted intensity and $I_{0}$ is the unattenuated beam intensity) and magnetic scattering intensity at the Mn- $\mathrm{L}_{3}$ edge, produced by small-angle X-ray scattering measurements. (d) X-ray absorption spectra and magnetic scattering intensity at the Ni- $\mathrm{L}_{3}$ edge, produced by small-angle X-ray scattering measurements.

ordering on the $\mathrm{Mn}$ and $\mathrm{Ni}$ sites in $\mathrm{MnNiGa}$, allowing future studies to more accurately model such systems.

The difference in X-ray wavelengths corresponding to the two absorption edges also causes an increased spatial resolution in the $\mathrm{Ni}$ image, by a scale factor of $E_{\mathrm{Ni}} / E_{\mathrm{Mn}}=$ $852 / 636 \approx 1.33$. A spatial resolution of $23 \pm 4 \mathrm{~nm}$ was achieved at the $\mathrm{Mn}-\mathrm{L}_{3}$ edge and $17 \pm 4 \mathrm{~nm}$ at the $\mathrm{Ni}-\mathrm{L}_{3}$ edge. Despite the greater spatial resolution at the Ni edge, the larger magnetic moment of $\mathrm{Mn}$ results in a greater scattering intensity, producing a more favorable signal-to-noise ratio in the reconstructed holographic images. Therefore, all following images were produced at the $\mathrm{Mn}-\mathrm{L}_{3}$ edge.

Figure 4 shows the sample in an increasing out-of-plane magnetic field. These images were collected by zero field cooling from above the Curie temperature $\left(T_{\mathrm{c}}=340 \pm 5 \mathrm{~K}\right)$, to $300 \mathrm{~K}$, and then increasing the magnetic field at fixed temperature. The first image was collected at zero field and shows a series of stripe domains with a periodicity of $275 \pm 6$ $\mathrm{nm}$ (Figure 4a). In uniaxial magnets the stripe period is typically determined by a balance of the domain wall energy 

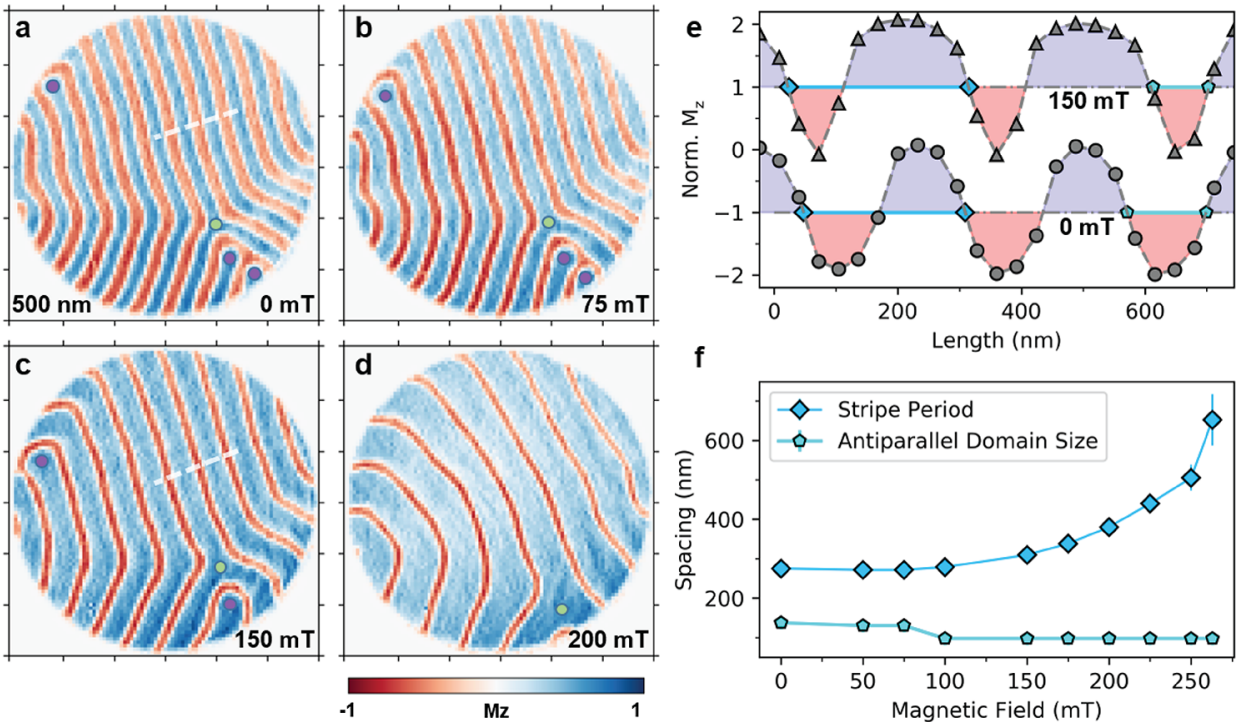

Figure 4. Field evolution of magnetic stripe domains. (a-d) Holographic images showing the evolution of a zero field cooled state of magnetic stripes evolving under an increasing out-of-plane magnetic field at $0,75,150$, and $200 \mathrm{mT}$, respectively. Holograms were produced at normal incidence to the sample. Positive and negative disclinations in the state are marked with purple and green circles. (e) Line profiles taken from the regions indicated by dotted lines in holograms (a) and (c). Circular markers indicate $0 \mathrm{mT}$, and triangular markers indicate $150 \mathrm{mT}$. Profiles are offset by 1 for clarity. Error bars are not visible on this scale. (f) Evolution of the stripe period and antiparallel domain size as a function of the out-of-plane magnetic field. These spacings were measured between the corresponding markers shown on panel (e) and similarly for other fields.

and the local demagnetizing energy. ${ }^{35}$ The observed stripe period here is within the range of length scales expected for the corresponding energies and is therefore consistent with MnNiGa behaving as a conventional uniaxial ferromagnet. ${ }^{25}$ The stripe phase here is also characterized by a zero net magnetization at remanence and, hence, zero global demagnetizing field. Figure $4 \mathrm{e}$ shows a line profile of this state taken orthogonal to the stripe direction, from the region marked by a dashed line in Figure 4a. In bulk uniaxial magnets the profile of a $180^{\circ}$ Bloch wall is given by $M_{z}(\mathbf{r})=M_{s} \tanh \left(\mathbf{r} / \delta_{0}\right)$, where $\mathbf{r}$ is the vector distance from the center of the wall, $M_{\mathrm{s}}$ is the saturation magnetization, and $\delta_{0}$ is the characteristic domain wall width, given by $\delta_{0}=\sqrt{\frac{A}{K_{u}}}$, where $A$ is the exchange stiffness. ${ }^{36}$ In samples with significant shape anisotropy, this profile is modified due to magnetostatic interactions; however, it is not possible to distinguish such models with the attained resolution. ${ }^{37}$ The profile is consistent with a repeating hyperbolic tangent domain wall size of $47 \pm 5 \mathrm{~nm}$.

As the out-of-plane magnetic field is applied to the sample, domains parallel to the magnetic field favor expansion, while the domains antiparallel favor contraction. Figure $4 \mathrm{e}$ shows the line profile of the stripe structure at $150 \mathrm{mT}$; there is a clear expansion of the parallel domains, forming solitonic domains akin to the helical distortions observed in helimagnets. ${ }^{38}$ As the domain walls retain their characteristic length, it is not possible for the antiparallel stripe domains to continuously shrink to arbitrary thicknesses, which forces a change in the periodicity of the system as the parallel domains grow. This is summarized in Figure 4f, as the antiparallel domain size undergoes a steplike reduction at $100 \mathrm{mT}$ and then remains constant within error as the stripe period grows. The corresponding measurements are marked on Figure 4e.

In finite size magnetic films the existence of an energy barrier between stripe states with differing numbers of domains forbids a continuous change in the period of the stripes. ${ }^{39}$ For ordered stripe domains this induces tension in the magnetic state, which can relax in the form of a kink or translational instability. However, the presence of magnetic dislocation defects greatly modifies this behavior. Dikshtein et al. argue that magnetic dislocations, which disrupt translational order, increase the entropy of the system, and at finite temperatures their nucleation, annihilation, and translation facilitate the relaxation of the stripe state in uniaxial magnets under changing fields and temperatures. ${ }^{40}$ Figure $4 \mathrm{a}$ shows the existence of three positive disclinations, where a stripe ends and is wrapped by the surrounding domain, and one negative disclination, where three different stripe orientations meet. These are indicated by purple and green circles, respectively. The angles subtended by the domains projecting from the negative disclination are $136 \pm 5^{\circ}, 142 \pm 5^{\circ}$, and $80 \pm 5^{\circ}$, suggesting these directions are not coupled to the hexagonal structure of $\mathrm{MnNiGa}$, where we would expect subtended angles in multiples of $60^{\circ}$. As the global state evolves under increasing magnetic field, there is a reduction in the number of disclinations, as they annihilate or move beyond the field of view. Such entities are topological defects, and it should be noted that while such dislocations can exist in the helical states of archetypal skyrmion-hosting systems, they have not been observed to mediate domain-based phase transitions in such systems. $^{41,42}$

As the field is increased beyond $200 \mathrm{mT}$, the stripe domains seen in Figure 4 evolve into magnetic bubbles, as shown in Figure 5. The breaking behavior exhibited, where a stripe splits, forming two separate domains, can be considered as a topological process as each break locally forms two $N=1 / 2$ topological structures, which cap the newly formed domains. ${ }^{43}$ These caps are observed to be thicker in radius than the remaining stripe, and this is likely a result of the domain wall growing to reduce its curvature which would otherwise impose a high energy cost. As the antiparallel domains contract they form elliptical bubbles and ultimately circular domains, which 

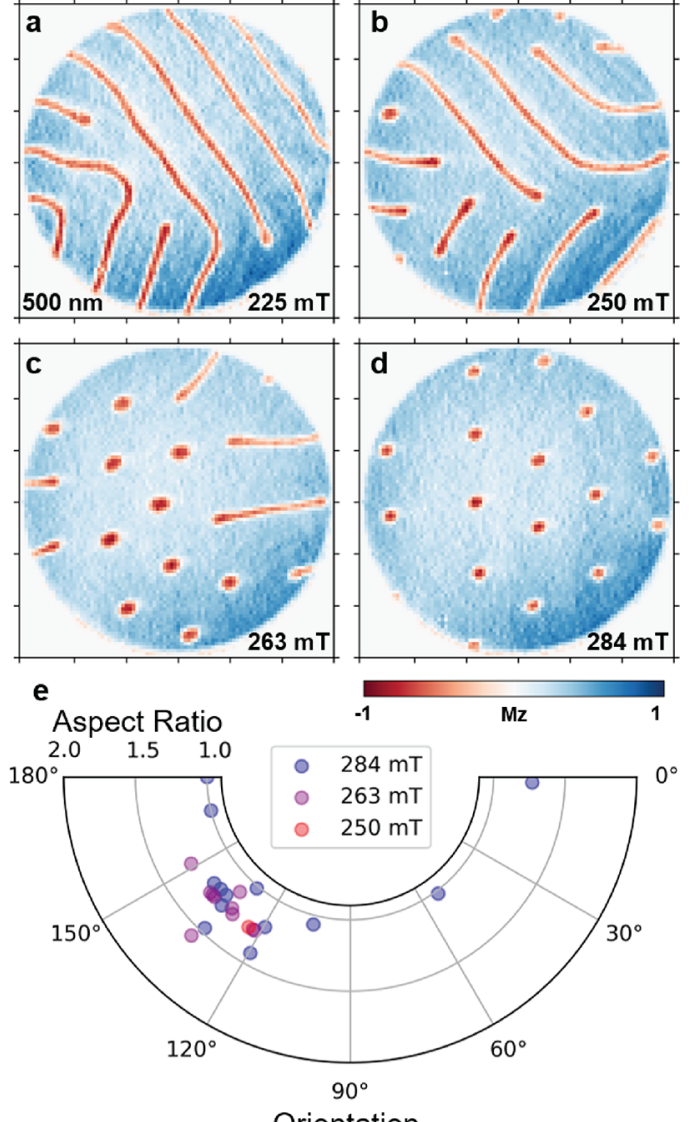

Orientation

Figure 5. Continued evolution of the stripe domains as a function of the magnetic field. (a-d) Holographic images of stripes rupturing and forming magnetic bubble domains at 225, 250, 263, and $284 \mathrm{mT}$. Holograms were produced at normal incidence to the sample. (e) Radial plot showing the aspect ratio and long axis orientation of the elliptical bubbles formed as a function of the out-of-plane magnetic field.

we identify as magnetic bubbles, as seen in Figure 5c. These bubbles are formed of two $N=1 / 2$ caps, making the isolated domain topologically identical to an $N=1$ skyrmion, in the case the caps are of the same chirality, and an $N=0$ type-II bubble, in the case the caps have opposite chirality. Significantly, these bubbles exhibit a single core domain structure, and while this is consistent with type-I and type-II magnetic bubble domains, it is not indicative of a biskyrmion texture. Theoretical models of uniaxial ferromagnets predict that, above a critical applied field, a first-order phase transition from stripes to a hexagonally ordered single core bubble state occurs. $^{35}$ Our observations indicate magnetic behavior consistent with a conventional uniaxial ferromagnet and not an exotic biskyrmion system.

Early experimental reports on $\mathrm{MnNiGa}$ also claimed that the biskyrmion/bubble density in a sample can be enhanced greatly by following a field cooling procedure. ${ }^{24}$ Figure $6 \mathrm{a}$ shows the resultant high density bubble state from field cooling with $87 \mathrm{mT}$ applied out-of-plane from above $T_{\mathrm{c}}$ to $300 \mathrm{~K}$ and then removing the field. The radius of bubbles in such a lattice expand with decreasing magnetic field, ${ }^{25}$ and at zero field the state also shows zero net magnetization within error. Figure $6 \mathrm{c}$ shows a magnified region of the sample, where a white tail persisting in a single direction between bubbles is visible. This has previously been discussed as evidence of an in-plane
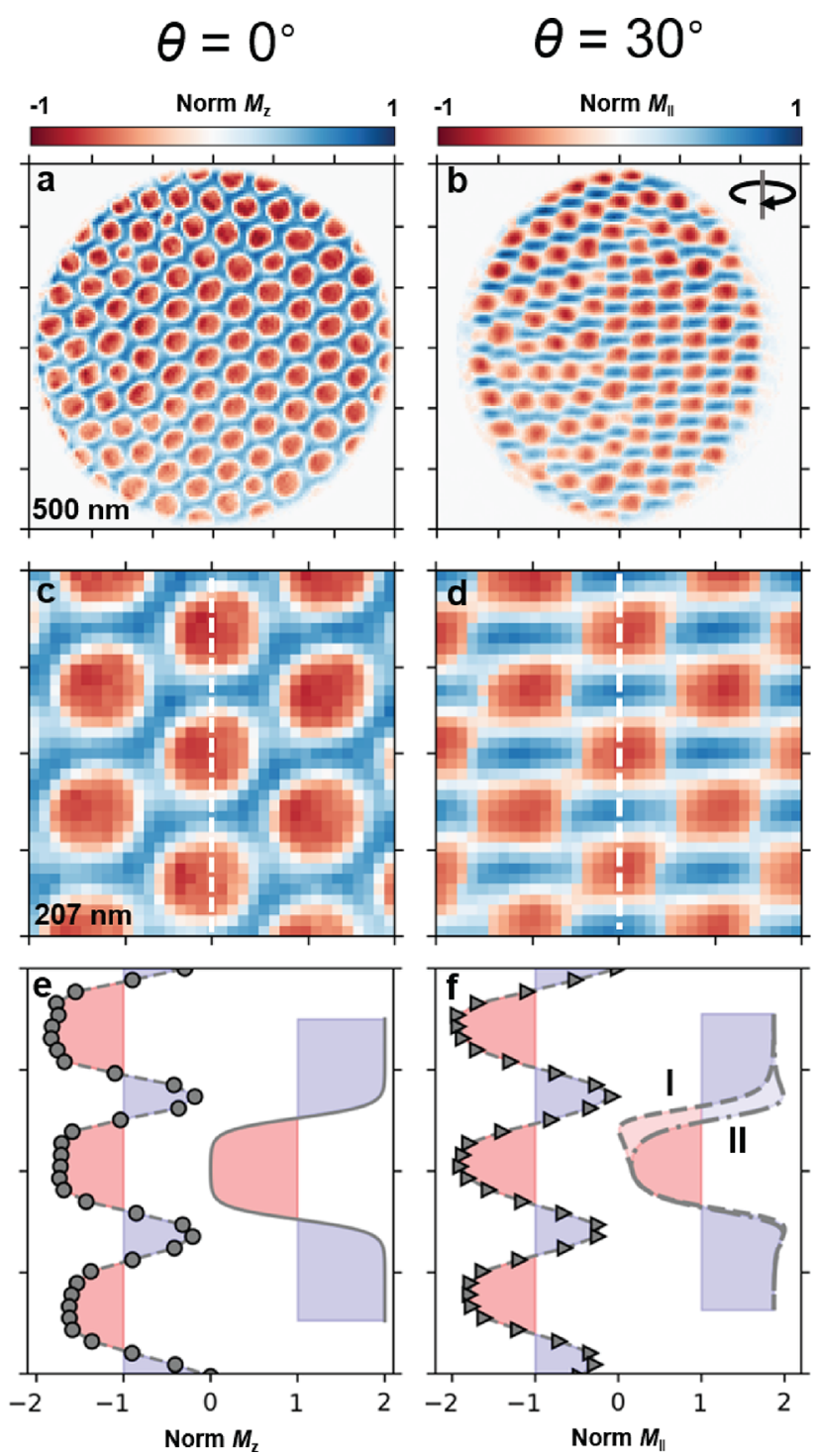

Figure 6. Normal and tilted imaging of the dense bubble lattice. (a) Holographic image of the field cooled bubble state at normal incidence. (b) Holographic image of the field cooled magnetic state projected after $30^{\circ}$ tilt about the vertical axis. (c) Magnified region of image (a). (d) Equivalent magnified region of the sample projected after $30^{\circ}$ tilt about the vertical axis. (e) Line profiles taken of the bubble projection in (c) following the white dashed lines in these figures. The left offset is the experimental data, and the right offset is the model profile for a single bubble. (f) Corresponding line profile of the magnetic bubbles at $30^{\circ}$ incidence is left offset, and line profile of the corresponding model type-I and type-II projections for a single bubble shown in Figure 2 are shown as dashed and dotted-dashed lines, respectively.

magnetization component, indicative of type-II bubbles. ${ }^{25}$ Figure 5e also shows there is a preferential orientation of the low density elliptical bubbles; however, the in-plane structure of the magnetic bubble's domain wall cannot be determined by $\mathrm{X}$-ray imaging at normal incidence.

We determined the complete structure of these bubbles by tilting the sample, in order to align components of the in-plane magnetization with the incoming beam. Tilting was performed on the zero field bubbles in order to prevent field perturbations to the state. Figure $6 \mathrm{~b}$ shows this state after tilting about the 

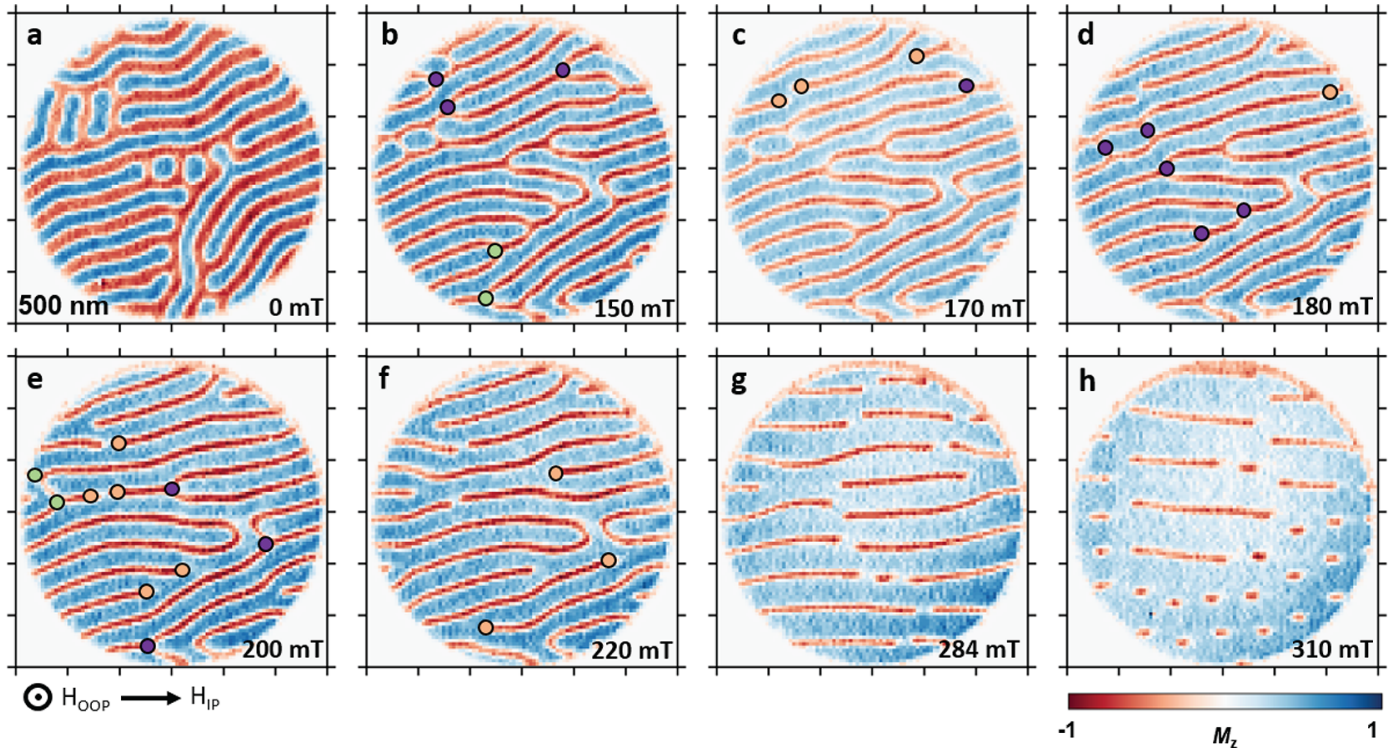

Figure 7. Evolution of a disordered stripe phase coexisting with several magnetic bubbles, in an increasing magnetic field applied $45^{\circ}$ face normal, with the in-plane component of the field in the right horizontal direction of the image. $(a-h)$ Holographic images with the applied magnetic field marked on each subfigure. The transformations of several magnetic dislocations are highlighted by colored circles, with purple marking the locations of dislocation before a transition and orange marking newly formed dislocations after a transition. Green circles mark pairs of dislocations in the frame preceding their annihilation. There are additional magnetic dislocations, which are not highlighted. Scale bar ticks indicate $500 \mathrm{~nm}$.

vertical image axis by $\theta=30^{\circ}$, and Figure $6 \mathrm{~d}$ shows the corresponding magnified region. Vertical line profiles taken from the respective magnified images shown in Figure 6e,f indicate there is a clear symmetric contraction of the bubble cores after tilting, consistent with the two domain walls of opposite chirality. This closely matches the inset model type-II projection taken from Figure 1 and is inconsistent with the type-I state. These model projections assume a magnetization texture which is uniform across the sample thickness and are calculated for $200 \mathrm{~nm}$ thick lamellae. It must be noted that while qualitative features such as the symmetric signal of a type-II bubble are retained, the exact details of such projections are highly dependent on the relative orientation of any anisotropic bubble to the tilt axis, in addition to the sample thickness; a more detailed analysis is given in the SI. It would be possible to further confirm the conclusions of these observations by tilting to $\theta=-30^{\circ}$ and observing a symmetric expansion of the type-II bubbles. These off-normal incident imaging methods point the way toward future tomographic and laminographic imaging in which the full three-dimensional magnetization vector field is reconstructed from a series of projections. $^{44}$

A further advantage of photon-based imaging techniques is the freedom of the sample environment; in contrast to electron-based techniques, electric and magnetic fields can be applied in any direction relative to the sample without deflecting the probe beam. ${ }^{45}$ Figure 7 shows the evolution of a disordered stripe and bubble phase at normal incidence, in an increasing magnetic field applied $45^{\circ}$ face normal, with the inplane component of the field in the right horizontal direction of the image. Under such conditions the out-of-plane field component causes domains with parallel magnetization to be energetically favored; however, the in-plane component of the field also causes it to be energetically favorable for in-plane magnetization structures, such as the domain walls, to align with that component of the applied field. This procedure has previously been used to preferentially produce type-II bubbles in amorphous $\mathrm{Fe} / \mathrm{Gd}$ multilayers, as it becomes energetically favorable for the in-plane magnetization on either side of a domain wall to align with the in-plane field component. ${ }^{46}$ The images show the expected expansion of blue domains, while the domain walls show a clear rotation toward horizontal ordering. As discussed above, the reorientation of the system is strongly mediated by the translation and transitioning of magnetic dislocations. Two types of dislocation transitions are observed: the annihilation of two defects and the transformation of one type of defect to another. Several instances of transforming dislocations are highlighted with colored circles, and each marked incidence represents a transition undergone by a red domain bifurcation. Green circles mark pairs of defects in the frame preceding their annihilation, and purple circles mark the location of a dislocation in the frame preceding its transition to a different type of dislocation, which is marked by orange in the following frame. A limited number of transitions have been highlighted for clarity. The lower region of Figure $7 \mathrm{~b}, \mathrm{c}$ shows the annihilation of two such defects, locally establishing longer ordered stripe domains. However, the most commonly observed type of transition is the breaking of a red domain bifurcation, forming a blue domain bifurcation. This process can also be considered as the formation of a $N=1 / 2$ domain cap on the red domains, as discussed above, which facilitates the transition of isolated blue bubble domains, to isolated red bubble domains. ${ }^{47}$ It should be noted that the observed field-induced reorientation of the stripe domains does not occur in the helical states of skyrmion hosting systems, and the formation of elliptical bubbles shown in Figure $7 \mathrm{~g}, \mathrm{~h}$ is also atypical of a skyrmionic system.

\section{CONCLUSIONS}

In conclusion, nanoscopic single core magnetic bubbles have been imaged within lamellae of $\mathrm{MnNiGa}$ using $\mathrm{X}$-ray holography. Tilted imaging was performed in order to measure 
the in-plane structure of these bubbles, which were shown to have a topologically trivial type-II structure. These measurements are inconsistent with the biskyrmion state that has been previously proposed. The evolution of stripe domains was tracked as a function of the magnetic field, with magnetic disclinations facilitating the relaxation of the striped state. These stripes ruptured above a critical field, forming single core magnetic bubble domains, consistent with previous work on conventional uniaxial magnets. By applying a magnetic field with both in- and out-of-plane field components, the evolution of a disordered stripe and bubble state was observed to be strongly mediated by local defects. This indicates the importance of such imaging studies in understanding the local properties of nanoscopic magnetic systems, if nanoscale topological magnetism is to be implemented effectively in advanced technologies.

\section{METHODS AND EXPERIMENTAL DETAILS}

Sample Synthesis. Polycrystalline samples of $\mathrm{MnNiGa}$ were prepared by arc-melting stoichiometric amounts of manganese (99.99\%, Sigma-Aldrich), nickel (99.9\%, Alfa Aesar) powders, and gallium shots $(99.99 \%$, Alfa Aesar) in an arc furnace under a $(5 \mathrm{~N})$ argon atmosphere. A $2 \%$ molar excess of $\mathrm{Mn}$ was added to compensate for losses during the arc-melting procedure. These buttons were annealed in a vacuum at $800^{\circ} \mathrm{C}$ for 1 week before being quenched in water to retain the high temperature structure. The phase purity of these samples was confirmed by X-ray diffraction using a Panalytical X-Pert Pro powder X-ray diffractometer operating in Bragg-Brentano geometry. This showed that the crystal was hexagonal $\mathrm{Pb}_{3} / \mathrm{mmc}$ with lattice parameters $a=b=4.15125(3) \AA$ and $c=5.32774(8) \AA$. The composition was determined to be $\mathrm{Mn}_{0.325(1)} \mathrm{Ni}_{0.324(1)} \mathrm{Ga}_{0.350(1)}$ by energy dispersive X-ray spectroscopy conducted using a Zeiss SUPRA 55-VP scanning electron microscope equipped with large area SDD EDX detector.

Sample Preparation. MnNiGa lamellae were prepared for this experiment using an FEI NanoLab600 Helios Dual-Beam focused ion beam (FIB) microscope equipped with an Omniprobe-200 micromanipulator. Samples of $10 \times 10 \mu \mathrm{m}^{2} \mathrm{MnNiGa}$ were extracted from a single grain using focused gallium ion-beam milling. The [001] easyaxis was within $7^{\circ}$ to face normal. The lamellae were thinned to 200 $\mathrm{nm}$ thickness and mounted over $3 \mathrm{~m}$ diameter sample apertures ion milled into $200 \mathrm{~nm} \mathrm{Si}{ }_{3} \mathrm{Ni}_{4}$ windows, coated with $600 \mathrm{~nm}$ of gold, as shown in Figure $1 \mathrm{~b}$. The gold was deposited by magnetron sputtering, with $20 \mathrm{~nm}$ thick chromium seed layers deposited for every $100 \mathrm{~nm}$ of gold. A reference slit of width $20 \mathrm{~nm}$ and length $6 \mu \mathrm{m}$ was cut $3.5 \mu \mathrm{m}$ from the center of the sample aperture, using the focused ion beam.

X-ray Holography. X-ray holography was undertaken on the COMET instrument at the SEXTANTS beamline at SOLEIL, using the holography with extended reference by autocorrelation linear differential operator (HERALDO) method. Magnetic contrast was generated by exploiting X-ray magnetic circular dichroism (XMCD) at the $\mathrm{L}_{3}$ absorption edge of $\mathrm{Mn}$ at $637 \mathrm{eV}$ and of $\mathrm{Ni}$ at $852 \mathrm{eV}$. Far field diffraction patterns formed via the interference of the sample and reference aperture were recorded using a $2048 \times 2048$ pixel chargecoupled device (CCD). The X-ray polarization was controlled with an HU44 APPLE II undulator.

\section{ASSOCIATED CONTENT}

\section{(s) Supporting Information}

The Supporting Information is available free of charge at https://pubs.acs.org/doi/10.1021/acsnano.0c07392.

X-ray holographic images of magnetic stripes and simulated X-ray projections of type II bubbles and biskyrmions as a function of orientation and thickness (PDF)

\section{AUTHOR INFORMATION}

\section{Corresponding Author}

Luke A. Turnbull - Centre for Materials Physics, Durham University, Durham DH1 3LE, United Kingdom; ○ orcid.org/0000-0001-5734-1154; Email: 1.a.turnbull@ durham.ac.uk

\section{Authors}

Max T. Birch - Centre for Materials Physics, Durham University, Durham DH1 3LE, United Kingdom; Diamond Light Source, Didcot OX11 ODE, United Kingdom

Angus Laurenson - School of Physics and Astronomy, University of Exeter, Exeter EX4 4QL, United Kingdom

Nick Bukin - School of Physics and Astronomy, University of Exeter, Exeter EX4 4QL, United Kingdom

Erick O. Burgos-Parra - Synchrotron SOLEIL, Saint Aubin 91192, France

Horia Popescu - Synchrotron SOLEIL, Saint Aubin 91192, France

Murray N. Wilson - Centre for Materials Physics, Durham University, Durham DH1 3LE, United Kingdom; (1) orcid.org/0000-0003-4731-646X

Aleš Stefančič - Department of Physics, University of Warwick, Coventry CV4 7AL, United Kingdom

Geetha Balakrishnan - Department of Physics, University of Warwick, Coventry CV4 7AL, United Kingdom

Feodor Y. Ogrin - School of Physics and Astronomy, University of Exeter, Exeter EX4 4QL, United Kingdom

Peter D. Hatton - Centre for Materials Physics, Durham University, Durham DH1 3LE, United Kingdom

Complete contact information is available at:

https://pubs.acs.org/10.1021/acsnano.0c07392

\section{Notes}

The authors declare no competing financial interest.

\section{ACKNOWLEDGMENTS}

This work was supported by the UK Skyrmion Project EPSRC Programme Grant (EP/N032128/1). We acknowledge SOLEIL for provision of synchrotron radiation facilities, and we would like to thank H. Popescu and N. Jaouen for assistance in using beamline SEXTANTS. We acknowledge Diamond Light Source for time on Beamline I10 under Proposal SI20866. M.N.W. acknowledges the support of the Natural Sciences and Engineering Research Council of Canada (NSERC).

\section{REFERENCES}

(1) Rößler, U.; Bogdanov, A.; Pfleiderer, C. Spontaneous Skyrmion Ground States in Magnetic Metals. Nature 2006, 442, 797-801.

(2) Iwasaki, J.; Mochizuki, M.; Nagaosa, N. Current-Induced Skyrmion Dynamics in Constricted Geometries. Nat. Nanotechnol. 2013, 8, 742-747.

(3) Yu, X.; Koshibae, W.; Tokunaga, Y.; Shibata, K.; Taguchi, Y.; Nagaosa, N.; Tokura, Y. Transformation between Meron and Skyrmion Topological Spin Textures in a Chiral Magnet. Nature 2018, 564, 95-98.

(4) Everschor-Sitte, K.; Masell, J.; Reeve, R. M.; Kläui, M. Perspective: Magnetic Skyrmions-Overview of Recent Progress in an Active Research Field. J. Appl. Phys. 2018, 124, 240901.

(5) Mühlbauer, S.; Binz, B.; Jonietz, F.; Pfleiderer, C.; Rosch, A.; Neubauer, A.; Georgii, R.; Böni, P. Skyrmion Lattice in a Chiral Magnet. Science 2009, 323, 915-919.

(6) Nagaosa, N.; Tokura, Y. Topological Properties and Dynamics of Magnetic Skyrmions. Nat. Nanotechnol. 2013, 8, 899-911. 
(7) Thiele, A. The Theory of Cylindrical Magnetic Domains. Bell Syst. Tech. J. 1969, 48, 3287-3335.

(8) Thiele, A. Device Implications of the Theory of Cylindrical Magnetic Domains. Bell Syst. Tech. J. 1971, 50, 725-773.

(9) Yu, X.; Mostovoy, M.; Tokunaga, Y.; Zhang, W.; Kimoto, K.; Matsui, Y.; Kaneko, Y.; Nagaosa, N.; Tokura, Y. Magnetic Stripes and Skyrmions with Helicity Reversals. Proc. Natl. Acad. Sci. U. S. A. 2012, 109, 8856-8860.

(10) Han, M.-G.; Garlow, J. A.; Liu, Y.; Zhang, H.; Li, J.; DiMarzio, D.; Knight, M. W.; Petrovic, C.; Jariwala, D.; Zhu, Y. Topological Magnetic-Spin Textures in Two-Dimensional van der Waals $\mathrm{Cr}_{2} \mathrm{Ge}_{2} \mathrm{Te}_{6}$. Nano Lett. 2019, 19, 7859-7865.

(11) Kurushima, K.; Tanaka, K.; Nakajima, H.; Mochizuki, M.; Mori, S. Microscopic Magnetization Distribution of Bloch Lines in a Uniaxial Magnet. J. Appl. Phys. 2019, 125, No. 053902.

(12) Chen, G. Skyrmion Hall Effect. Nat. Phys. 2017, 13, 112-113.

(13) Peng, L.; Zhang, Y.; He, M.; Ding, B.; Wang, W.; Tian, H.; Li, J.; Wang, S.; Cai, J.; Wu, G.; Liu, J. P.; Kramer, M. J.; Shen, B. Generation of High-Density Biskyrmions by Electric Current. npj Quantum Mater. 2017, 2, 30.

(14) Hou, Z.; Zhang, Q.; Xu, G.; Zhang, S.; Gong, C.; Ding, B.; Li, H.; Xu, F.; Yao, Y.; Liu, E.; Wu, G.; Zhang, X.-x.; Wang, W. Manipulating the Topology of Nanoscale Skyrmion Bubbles by Spatially Geometric Confinement. ACS Nano 2019, 13, 922-929.

(15) Li, Z.-A.; Zheng, F.; Tavabi, A. H.; Caron, J.; Jin, C.; Du, H.; Kovács, A.; Tian, M.; Farle, M.; Dunin-Borkowski, R. E. Magnetic Skyrmion Formation at Lattice Defects and Grain Boundaries Studied by Quantitative off-Axis Electron Holography. Nano Lett. 2017, 17, $1395-1401$

(16) Yu, X.; Kanazawa, N.; Onose, Y.; Kimoto, K.; Zhang, W.; Ishiwata, S.; Matsui, Y.; Tokura, Y. Near Room-Temperature Formation of a Skyrmion Crystal in Thin-Films of the Helimagnet FeGe. Nat. Mater. 2011, 10, 106-109.

(17) Tonomura, A.; Yu, X.; Yanagisawa, K.; Matsuda, T.; Onose, Y.; Kanazawa, N.; Park, H. S.; Tokura, Y. Real-Space Observation of Skyrmion Lattice in Helimagnet $\mathrm{MnSi}$ Thin Samples. Nano Lett. 2012, 12, 1673-1677.

(18) Park, H. S.; Yu, X.; Aizawa, S.; Tanigaki, T.; Akashi, T.; Takahashi, Y.; Matsuda, T.; Kanazawa, N.; Onose, Y.; Shindo, D.; Tonomura, A.; Tokura, Y. Observation of the Magnetic Flux and Three-Dimensional Structure of Skyrmion Lattices by Electron Holography. Nat. Nanotechnol. 2014, 9, 337-342.

(19) Mathur, N.; Stolt, M. J.; Niitsu, K.; Yu, X.; Shindo, D.; Tokura, Y.; Jin, S. Electron Holography and Magnetotransport Measurements Reveal Stabilized Magnetic Skyrmions in Fe1-xCoxSi Nanowires. ACS Nano 2019, 13, 7833-7841.

(20) Phatak, C.; Petford-Long, A.; De Graef, M. Recent Advances in Lorentz Microscopy. Curr. Opin. Solid State Mater. Sci. 2016, 20, 107-114.

(21) Yu, X.; Onose, Y.; Kanazawa, N.; Park, J.; Han, J.; Matsui, Y.; Nagaosa, N.; Tokura, Y. Real-Space Observation of a TwoDimensional Skyrmion Crystal. Nature 2010, 465, 901-904.

(22) Yu, X.; Tokunaga, Y.; Kaneko, Y.; Zhang, W.; Kimoto, K.; Matsui, Y.; Taguchi, Y.; Tokura, Y. Biskyrmion States and Their Current-Driven Motion in a Layered Manganite. Nat. Commun. 2014, 5,3198 .

(23) Wang, W.; Zhang, Y.; Xu, G.; Peng, L.; Ding, B.; Wang, Y.; Hou, Z.; Zhang, X.; Li, X.; Liu, E.; Wang, S.; Cai, J.; Wang, F.; Li, J.; Hu, F.; Wu, G.; Shen, B.; Zhang, X.-X. A Centrosymmetric Hexagonal Magnet with Superstable Biskyrmion Magnetic Nanodomains in a Wide Temperature Range of 100-340 K. Adv. Mater. 2016, 28, 6887-6893.

(24) Peng, L.; Zhang, Y.; Wang, W.; He, M.; Li, L.; Ding, B.; Li, J.; Sun, Y.; Zhang, X.-G.; Cai, J.; Wang, S.; Wu, G.; Shen, B. Real-Space Observation of Nonvolatile Zero-Field Biskyrmion Lattice Generation in MnNiGa Magnet. Nano Lett. 2017, 17, 7075-7079.

(25) Loudon, J. C.; Twitchett-Harrison, A. C.; Cortés-Ortuño, D.; Birch, M. T.; Turnbull, L. A.; Stefančič, A.; Ogrin, F. Y.; Burgos-Parra, E. O.; Bukin, N.; Laurenson, A.; Popescu, H.; Beg, M.; Hovorka, O.;
Fangohr, H.; Midgley, P.; Balakrishnan, G.; Hatton, P. D. Do Images of Biskyrmions Show Type-II Bubbles? Adv. Mater. 2019, 31, 1806598.

(26) Yao, Y.; Ding, B.; Cui, J.; Shen, X.; Wang, Y.; Wang, W.; Yu, R. Magnetic Hard Nanobubble: A Possible Magnetization Structure behind the Bi-Skyrmion. Appl. Phys. Lett. 2019, 114, 102404.

(27) Blume, M.; Gibbs, D. Polarization Dependence of Magnetic XRay Scattering. Phys. Rev. B: Condens. Matter Mater. Phys. 1988, 37, $1779-1789$

(28) Van der Laan, G.; Figueroa, A. I. X-Ray Magnetic Circular Dichroism - A Versatile Tool to Study Magnetism. Coord. Chem. Rev. 2014, 277, 95-129.

(29) Eisebitt, S.; Lüning, J.; Schlotter, W.; Lörgen, M.; Hellwig, O.; Eberhardt, W.; Stöhr, J. Lensless Imaging of Magnetic Nanostructures by X-Ray Spectro-Holography. Nature 2004, 432, 885-888.

(30) Zhu, D.; Guizar-Sicairos, M.; Wu, B.; Scherz, A.; Acremann, Y.; Tyliszczak, T.; Fischer, P.; Friedenberger, N.; Ollefs, K.; Farle, M.; Fienup, J. R.; Stöhr, J. High-Resolution X-Ray Lensless Imaging by Differential Holographic Encoding. Phys. Rev. Lett. 2010, 105, No. 043901.

(31) Ukleev, V.; Yamasaki, Y.; Morikawa, D.; Karube, K.; Shibata, K.; Tokunaga, Y.; Okamura, Y.; Amemiya, K.; Valvidares, M.; Nakao, H.; Taguchi, Y.; Tokura, Y.; Arima, T. Element-Specific Soft X-Ray Spectroscopy, Scattering, and Imaging Studies of the SkyrmionHosting Compound $\mathrm{Co}_{8} \mathrm{Zn}_{8} \mathrm{Mn}_{4}$. Phys. Rev. B: Condens. Matter Mater. Phys. 2019, 99, 144408.

(32) Duckworth, T. A.; Ogrin, F.; Dhesi, S. S.; Langridge, S.; Whiteside, A.; Moore, T.; Beutier, G.; van der Laan, G. Magnetic Imaging by X-Ray Holography Using Extended References. Opt. Express 2011, 19, 16223-16228.

(33) Bukin, N.; Burgos-Parra, C. M. E.; Keatley, P. S.; Hicken, R. J.; Ogrin, F. Y.; Beutier, G.; Dupraz, M.; Popescu, H.; Jaouen, N.; Yakhou-Harris, F.; Cavill, S. A.; van der Laan, G.; McKeever, C. TimeResolved Imaging of Magnetic Vortex Dynamics Using Holography with Extended Reference by Autocorrelation Linear Differential Operator. Sci. Rep. 2016, 6, 36307.

(34) Guizar-Sicairos, M.; Fienup, J. R. Holography with Extended Reference by Autocorrelation Linear Differential Operation. Opt. Express 2007, 15, 17592-17612.

(35) Garel, T.; Doniach, S. Phase Transitions with Spontaneous Modulation - The Dipolar Ising Ferromagnet. Phys. Rev. B: Condens. Matter Mater. Phys. 1982, 26, 325-328.

(36) Skomski, R. Length Scales in Magnetism, Encyclopedia of Materials: Science and Technology, 2nd ed.; Elsevier: Oxford, 2006; Vol. 1; pp 1-7.

(37) Hubert, A.; Schafer, R. Magnetic Domains: The Analysis of Magnetic Microstructures, 1st ed.; Springer Verlag: Berlin, Heidelberg, 1998; Vol. 1; pp 100-120.

(38) Wilson, M. N.; Karhu, E. A.; Lake, D. P.; Quigley, A. S.; Meynell, S.; Bogdanov, A. N.; Fritzsche, H.; Rößler, U. K.; Monchesky, T. L. Discrete Helicoidal States in Chiral Magnetic Thin Films. Phys. Rev. B: Condens. Matter Mater. Phys. 2013, 88, 214420 .

(39) Dikshtein, I.; Lisovskii, F.; Mansvetova, E.; Tarasenko, V. Magnetic Dislocations in a Stripe Domain Structure. Sov. Phys. JETP 1990, 71, 1213-1223.

(40) Dikshtein, I.; Lisovskii, F.; Mansvetova, E.; Tarasenko, V. Types of Instability in Ordered Domains Structures. Sov. Phys. JETP 1991, $73,114-124$

(41) Uchida, M.; Onose, Y.; Matsui, Y.; Tokura, Y. Real-Space Observation of Helical Spin Order. Science 2006, 311, 359-361.

(42) Matsumoto, T.; So, Y.-G.; Kohno, Y.; Sawada, H.; Ikuhara, Y.; Shibata, N. Direct Observation of $\Sigma 7$ Domain Boundary Core Structure in Magnetic Skyrmion Lattice. Sci. Adv. 2016, 2, e1501280.

(43) Ezawa, M. Compact Merons and Skyrmions in Thin Chiral Magnetic Films. Phys. Rev. B: Condens. Matter Mater. Phys. 2011, 83, 100408 .

(44) Donnelly, C.; Finizio, S.; Gliga, S.; Holler, M.; Hrabec, A.; Odstrčil, M.; Mayr, S.; Scagnoli, V.; Heyderman, L.; Raabe, M. G.-S. 
J.; Guizar-Sicairos, M. Time-Resolved Imaging of Three-Dimensional Nanoscale Magnetization Dynamics. Nat. Nanotechnol. 2020, 15, 356-360.

(45) Birch, M. T.; Cortés-Ortuño, D.; Turnbull, L. A.; Wilson, M. N.; Groß, F.; Träger, N.; Laurenson, A.; Bukin, N.; Moody, S. H.; Weigand, M.; Schütz, G.; Popescu, H.; Fan, R.; Steadman, P.; Verezhak, J. A. T.; Balakrishnan, G.; Loudon, J. C.; TwitchettHarrison, A. C.; Hovorka, O.; Fangohr, H.; et al. Real-Space Imaging of Confined Magnetic Skyrmion Tubes. Nat. Commun. 2020, 11, 1726.

(46) Je, S.-G.; Han, H.-S.; Kim, S. K.; Montoya, S. A.; Chao, W.; Hong, I.-S.; Fullerton, E. E.; Lee, K.-S.; Lee, K.-J.; Im, M.-Y.; Hong, J.I. Direct Demonstration of Topological Stability of Magnetic Skyrmions via Topology Manipulation. ACS Nano 2020, 14, 32513258.

(47) Pamyatnykh, L.; Filippov, B.; Agafonov, L.; Lysov, M. Motion and Interaction of Magnetic Dislocations in Alternating Magnetic Field. Sci. Rep. 2017, 7, 18084. 